\title{
Emodin induces Panc-1 cell apoptosis via declining the mitochondrial membrane potential
}

\author{
JIN-XIANG LIU ${ }^{1 *}$, JIAN-HONG ZHANG $^{1 *}$, HONG-HAI LI $^{1 *}$, FU-JI LAI $^{1}$, KANG-JIE CHEN ${ }^{2}$, \\ HUI CHEN ${ }^{1}$, JIANG LUO ${ }^{1}$, HONG-CHUN GUO ${ }^{1}$, ZHAO-HONG WANG ${ }^{1}$ and SHENG-ZHANG LIN ${ }^{1,2}$ \\ ${ }^{1}$ Department of Hepatobiliary-Pancreatic Surgery, The Second Affiliated Hospital of Wenzhou Medical College, \\ Wenzhou 325027; ${ }^{2}$ Department of Hepatobiliary-Pancreatic Surgery, First Affiliated Hospital, \\ Zhejiang University, School of Medicine, Hangzhou 310003, P.R. China
}

Received June 13, 2012; Accepted July 12, 2012

DOI: $10.3892 /$ or.2012.2042

\begin{abstract}
In this study, we investigated the apoptotic effect of emodin on human pancreatic cancer cell line Panc-1 in vitro and in vivo as well as the possible mechanisms involved. In vitro, human pancreatic cancer cell line Panc-1 was exposed to varying concentrations of emodin $(0,10,20,40$ or $80 \mu \mathrm{mol} / \mathrm{l})$. Then the mitochondrial membrane potential (MMP) was analyzed by JC-1 staining, cell apoptosis was analyzed by flow cytometry (FCM) and cell proliferation was analyzed by MTT. In vivo, nude mice orthotopically implanted were randomly divided into five groups to receive treatments by different doses of emodin: control group (normal saline $0.2 \mathrm{ml}$ ), $\mathrm{E}_{10}$ group (emodin $10 \mathrm{mg} / \mathrm{kg}$ ), $\mathrm{E}_{20}$ group (emodin $20 \mathrm{mg} / \mathrm{kg}$ ), $\mathrm{E}_{40}$ group (emodin $40 \mathrm{mg} / \mathrm{kg}$ ) and $\mathrm{E}_{80}$ group (emodin $80 \mathrm{mg} / \mathrm{kg}$ ). Each mouse was treated 5 times by intraperitoneal injection of emodin every 3 days. During the treatment, the feeding stuff was recorded. One week after the last treatment, we recorded the body weight and the maximum diameter of tumor in each group before the mice were sacrificed. Then the cell apoptosis of the tumor was tested by TUNEL assay. The results in vitro showed that the MMP of the cells declined and the apoptosis rate increased with the emodin concentration increasing and the cell proliferation of each group was inhibited in a dose- and time-dependent manner by emodin. The feeding stuff curve did not decline significantly in $\mathrm{E}_{40}$ group and the apoptosis rate of the tumor cells in this group was higher than the lower-dose groups. Taken together, our results demonstrate that emodin may induce the pancreatic cancer cell apoptosis via declining the MMP and a moderate dose of emodin improved the living state of the model mice.
\end{abstract}

Correspondence to: Dr Sheng-Zhang Lin, Department of Hepatobiliary-Pancreatic Surgery, The Second Affiliated Hospital of Wenzhou Medical College, 109 Xue-yuan Road, Wenzhou 325027, P.R. China

E-mail: wzf21sz@163.com

*Contributed equally

Key words: emodin, pancreatic cancer, mitochondrial membrane potential

\section{Introduction}

Pancreatic cancer is known for its high mortality rate. It is one of the most common causes of cancer mortality in developed countries (1). Due to the lack of effective-specific diagnosis methods, $26 \%$ of patients are in the middle-late stage when being diagnosed and only $<10 \%$ of patients has a surgery opportunity, and even if the patients have the operation, they still need a series of comprehensive therapies, including chemotherapy (2). In the past decades, gemcitabine was prefered as the first-line drug for pancreatic cancer chemotherapy, but the benefits were very limited, so that new chemotherapy regimens for pancreatic cancer were explored and tested. But new chemotherapy results did not achieve considerable achievement and still could not replace gemcitabine as the gold standard for clinical treatment (3). Thus, it is extremely urgent to look for agents that have low toxicity and highefficency.

Emodin, 1,3,8-trihydroxyl-6-methyl anthraquinone, is a monomer of Chinese Herb separated from Rhubarb Genera and Polygonum and Rhamnus and Folium Sennae. A number of studies show that emodin has a good effect on treating prostate (4), colorectal (5) and pancreatic cancer (6). In this study, we found that the Panc- 1 cells' MMP declined and apoptosis rate increased dose-dependently with emodin treatment and the cell proliferation of each group was inhibited in a dose-and time-dependent manner. The feeding stuff curve did not decline significantly in the group of the mice treated with emodin at the dose of $40 \mathrm{mg} / \mathrm{kg}$ and the apoptosis rate of the tumor cells was higher than the lower-dose groups. These results demonstrated that emodin could induce Panc-1 cells apoptosis via declining the MMP and a moderate dose of emodin improved the living state of the model mice.

\section{Materials and methods}

Chemicals and reagents. Emodin and MTT were purchased from Sigma-Aldrich (St. Louis, MO, USA). Emodin was dissolved in dimethyl sulfoxide (DMSO). The final concentration of DMSO used in vitro was $<0.1 \%$ and that in vivo $<1 \%$. Dulbecco's modified Eagle's medium (DMEM), fetal bovine serum (FBS), penicillin-streptomycin, trypsin-EDTA were 
obtained from Gibco-BRL (Invitrogen, Grand Island, NY, USA).

5,5',6,6'-tetrachloro-1,1',3,3'-tetraethylbenzimidazolcarbocyanine iodide (JC-1) kit was purchased from Beyotime Biotechnology (Haimen, China). Annexin V-FITC cell apoptosis detection kit was purchased from Nanjing KeyGen Biotechology Co., Ltd., (Nanjing, China). Terminal deoxynucleotidyl transferase-mediated deoxyuridine triphosphate nick-end labeling (TUNEL) kit was purchased from Roche Co. (Mannheim, Germany).

Cell line and cell culture. Human pancreatic cancer cell line Panc-1 was purchased from the American Type Culture Collection and cultured in DMEM with 10\% FBS, $100 \mathrm{units} / \mathrm{ml}$ penicillin, and $100 \mu \mathrm{g} / \mathrm{ml}$ streptomycin at $37^{\circ} \mathrm{C}$ under a humidified $5 \% \mathrm{CO}_{2}$ atmosphere. Cells were passaged at $80-90 \%$ confluence.

$J C-1$ analysis. Panc-1 cells were placed in 6-well plates and cultured overnight. Then, Panc-1 cells were cultured in the medium with $0,10,20,40$ or $80 \mu \mathrm{mol} / 1$ emodin for $24 \mathrm{~h}$. After that, Panc-1 cells were stained with $5 \mathrm{mg} / \mathrm{ml} \mathrm{JC}-1$ for $20 \mathrm{~min}$ at $37^{\circ} \mathrm{C}$ in the dark. Then MMP depletion was observed under a fluorescence microscope.

FCM analysis. Panc-1 cells $\left(4 \times 10^{5}\right)$ were seeded to each 6-well plate. When cells adhered to the culture flask wall, they were treated with $0,10,20,40$ or $80 \mu \mathrm{mol} / 1$ emodin for $24 \mathrm{~h}$ before collected for trypsin digestion. The cell apoptosis were then detected according to the instructions of Annexin V-FITC cell apoptosis detection kit and assessed by FCM (BectonDickinson, San Jose, CA, USA).

MTT analysis. Panc-1 cells were cultured in the medium with various concentrations of emodin $(0,10,20,40$ or $80 \mu \mathrm{mol} / \mathrm{l})$ for different time $(12,24,48$ or $72 \mathrm{~h})$ and then the viability of Panc-1 cells was determined by MTT. Briefly, cells were plated at a density of $5 \times 10^{3}$ cells/well in 96 -well microtiter plates. After treatment, $20 \mu \mathrm{l}$ of MTT solution $[5 \mathrm{mg} / \mathrm{ml}$ in phosphatebuffered saline (PBS)] was added to each well and the plates were incubated. The supernatant was aspirated and the MTT formazan was dissolved in $150 \mu \mathrm{l}$ of DMSO. The plates were mixed for $10 \mathrm{~min}$ on a gyratory shaker and absorbance was measured with an ELISA reader (Bio-Tek ELx800, Winooski, VT, USA) at a wavelength of $490 \mathrm{~nm}$. Experiment was repeated thrice. Inhibition rate $(\%)=[1-($ dosing absorbance - blank absorbance)/(control absorbance - blank absorbance)] x $100 \%$.

Experimental animals. Male nude mice [4-5 weeks old, BALB/c (nu/nu)), weight 17-18 g) were purchased from Shanghai Cancer Institute for Tumor Implantation and maintained in a specific pathogen free (SPF) environment in the Animal Experiment Center of the Wenzhou Medical College. All animal studies were approved by the Animal Research and Ethics Committee of the Wenzhou Medical College.

Model establishment and experiment scheme. Suspensions consisting of Panc-1 cells in serum-free medium, with $>90 \%$ viability, were used for model establishment. Mice were anesthetized with $2 \%$ pentobarbital sodium solution and a small left abdominal flank incision was made. Panc- 1 cells $\left(5 \times 10^{6}\right)$ in $100 \mu \mathrm{l}$ serum-free medium were injected into the subcapsular region of the pancreas using a 27-gauge needle. The abdominal wound was closed in one layer.

After three weeks, the model mice were divided into five groups randomly to receive different treatment: control group (N, physiological saline), $\mathrm{E}_{10}$ group (emodin, $10 \mathrm{mg} / \mathrm{kg}$ ), $\mathrm{E}_{20}$ group (emodin, $20 \mathrm{mg} / \mathrm{kg}$ ), $\mathrm{E}_{40}$ group (emodin, $40 \mathrm{mg} / \mathrm{kg}$ ), $\mathrm{E}_{80}$ group (emodin, $80 \mathrm{mg} / \mathrm{kg}$ ). Each mouse was treated 5 times by intraperitoneal injection of emodin every 3 days. The feeding stuff intake was recorded before every treatment. One week after the last treatment, the body weight was measured and the mice were euthanized with $2 \%$ sodium pentobarbital, followed by measuring the largest diameter of tumors. Finally, implanted tumors were formalin-fixed and paraffin-embedded for subsequent TUNEL assay.

TUNEL analysis. We assessed the degree of tumor apoptosis with the TUNEL assay after the nude mice were sacrificed. TUNEL staining of paraffin-embedded tumor sections was done with the TUNEL kit according to the instructions. Laser scanning confocal microscope (Olympus BX51, Japan) under 400 -fold observation camera was used, with excitation wavelength $488 \mathrm{~nm}$ and emission wavelength $568 \mathrm{~nm}$. We observed 10 field visions of the strongest fluorescence on each slice.

Statistical analysis. SPSS 17.0 was used for statistical analysis. Data are presented as the means \pm SD. Differences among groups of cells or mice were analyzed by one-way ANOVA followed by unpaired Student's t-test. $\mathrm{P}<0.05$ was considered statistically significant.

\section{Results}

Emodin induces MMP decline in human pancreatic cancer cell line Panc-1. JC-1 fluorescent dyes can gather in the matrix of mitochondria and produce red fluorescence. If the MMP is reduced, JC-1 can not gather the matrix so that JC-1 exists in the matrix as a monomer, producing green fluorescence. JC-1 fluorescent color changed from red to green along with the increasing concentration of emodin, suggesting that MMP declined along with the increasing concentration of emodin (Fig. 1).

Emodin induces apoptosis of human pancreatic cancer Panc-1 cells. We employed Annexin V-FITC cell apoptosis detection kit to detect cell apoptosis. Our study showed that cell apoptosis rate of each group was upregulated dose-dependently by emodin (Fig. 2).

Emodin inhibits the proliferation of human pancreatic cancer cell Panc-1. The cell proliferation-inhibition rate was detected by MTT assay. We found that cell proliferation was inhibited dose- and time-dependently. With treatment of $40 \mu \mathrm{mol} / 1$ emodin, the inhibition rate of each time point (12, 24,48 or $72 \mathrm{~h}$ ) is $3.66 \pm 0.99 \%, 17.67 \pm 0.49 \%, 38.13 \pm 0.11 \%$ and $54.73 \pm 0.83 \%$, respectively (Fig. 3).

Emodin increases the amount of feed of model mice. We recorded the feeding stuff eaten by each mouse each day. Data 

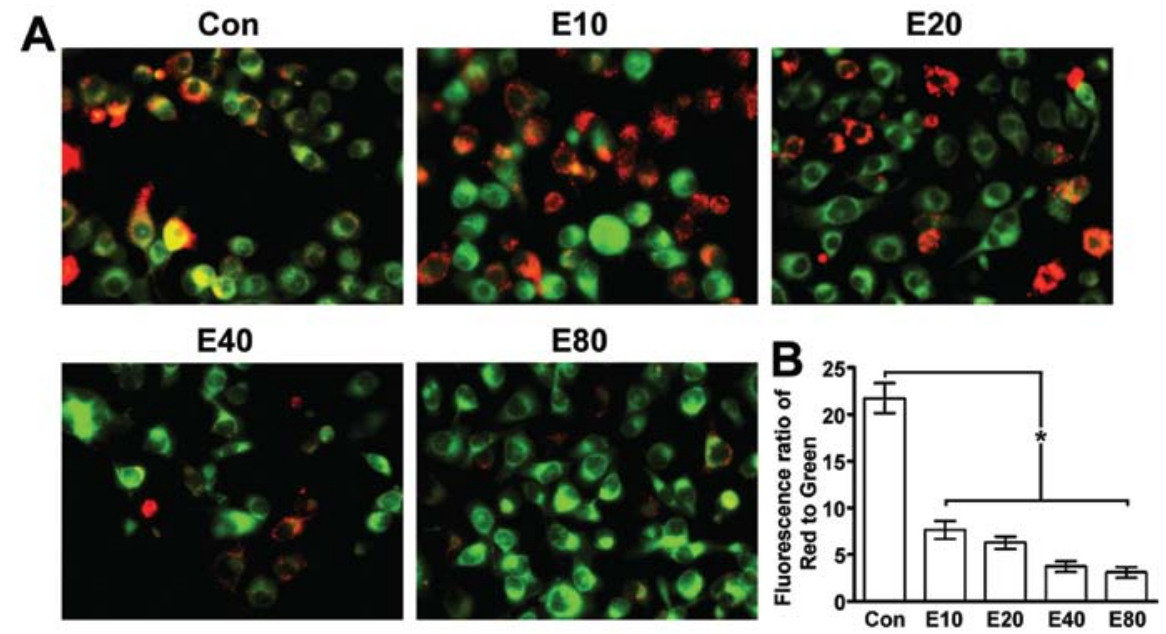

Figure 1. Effect of various concentrations of emodin on the MMP in Panc-1 cells. (A) Representative pictures are shown. JC-1 fluorescent dyes can gather in the matrix of mitochondria and produce red fluorescence. As the MMP declined, JC-1 can not gather the matrix so that JC-1 exist in the matrix as monomer, producing green fluorescence. (B) The ratio of the red (OD1) and green (OD2) optical density is shown. As the concentration of emodin increased, the ratio declined obviously. ${ }^{*} \mathrm{P}<0.05$, compared to the control group.
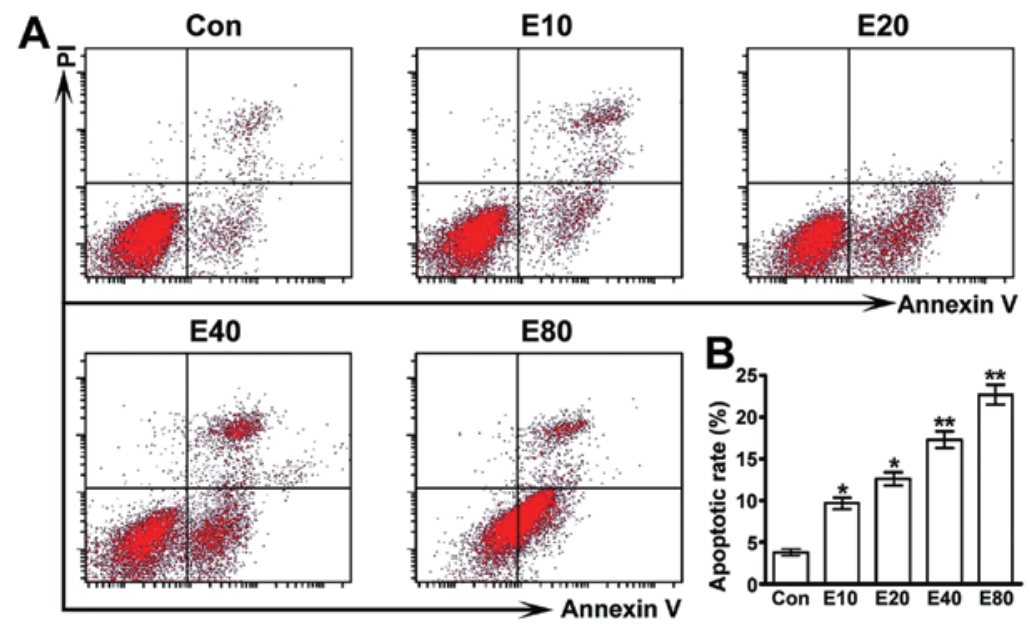

Figure 2. Effect of various concentrations of emodin on the apoptosis of Panc-1 cells. (A) Representative dot-plots illustrating the apoptotic status of Panc-1 cells. (B) The percentage of apoptotic Panc-1 cells. ${ }^{*} \mathrm{P}<0.05$, compared to the control group; ${ }^{* *} \mathrm{P}<0.01$, compared to the control group.

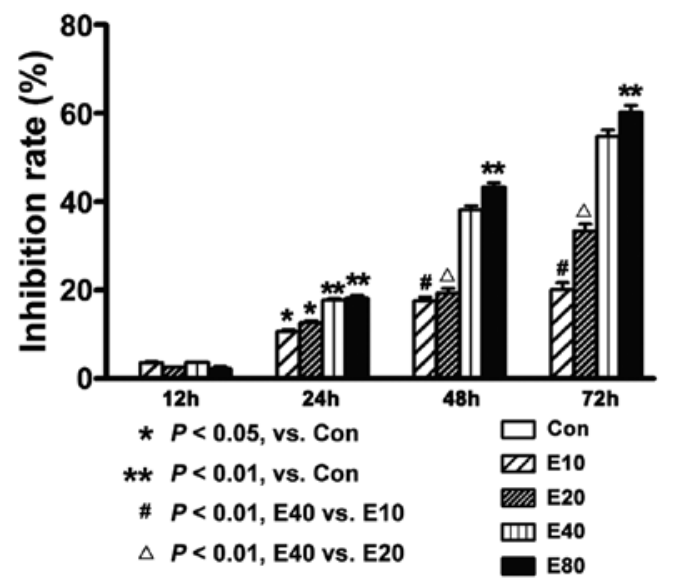

Figure 3. Effect of various concentrations of emodin on the proliferation of Panc-1 cells in vitro. The cells without drug treatment were used as the control. Emodin inhibited the proliferation of Panc-1 cells in a dose- and time-dependent manner. Data are expressed as the means $\pm \mathrm{SD} .{ }^{*} \mathrm{P}<0.05$, compared to the control group; ${ }^{* *} \mathrm{P}<0.01$, compared to the control group; ${ }^{\text {" }} \mathrm{P}<0.01$ and ${ }^{\Delta} \mathrm{P}<0.01$, compared to $40 \mu \mathrm{mol} / 1$ emodin treated cells.

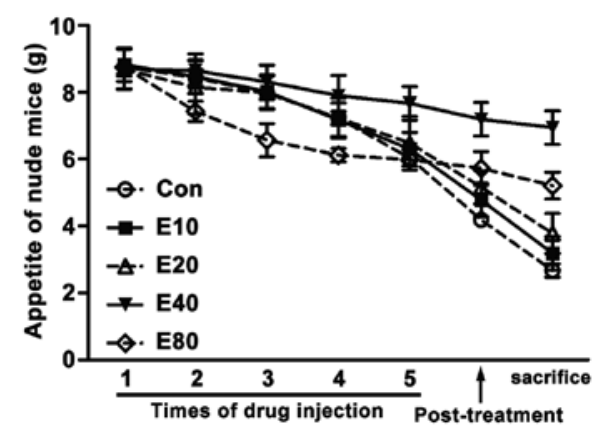

Figure 4. Effect of various concentrations of emodin on the feeding stuff eaten by nude mice. Feeding stuff that the mice of control group ate declined rapidly while the experimental group declined mildly. The curve of $\mathrm{E}_{80}$ group declined rapidly at the first stage, but at the last stage the curve become less than the control, $\mathrm{E}_{10}$ and $\mathrm{E}_{20}$ group.

showed that at the begining of experiment, feeding stuff intake of each group was equal. As treatment progressing, feeding 
A

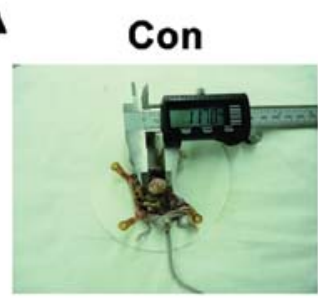

E40

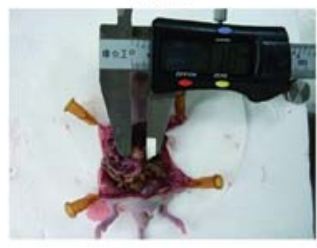

E10

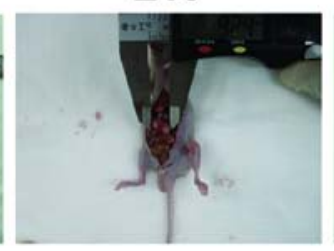

E80

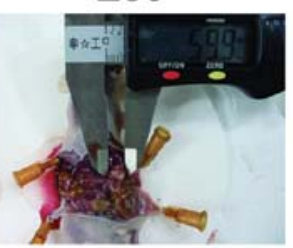

E20
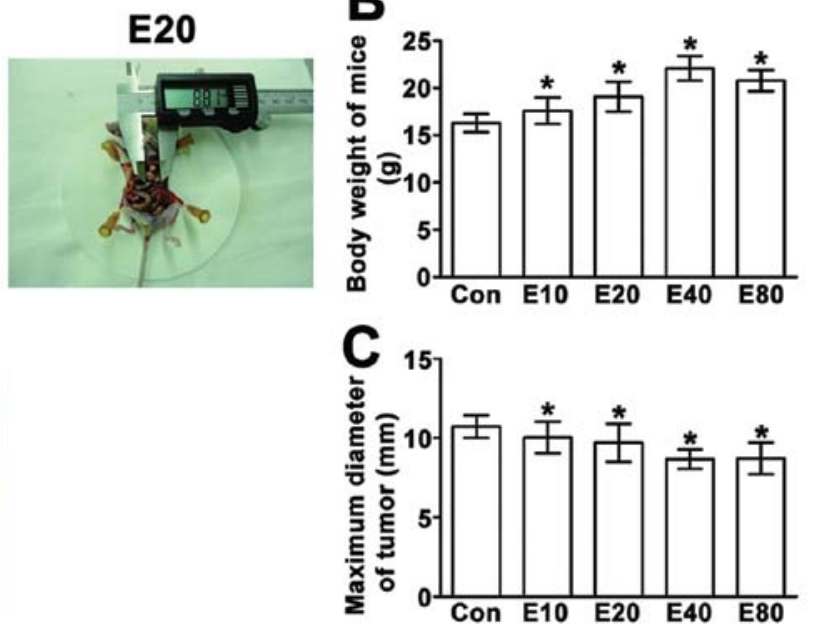

Figure 5. Effect of various concentrations of emodin on tumor growth and body weight of nude mice. (A) Images of orthotopically implanted pancreatic tumors one week after the last treatment. Emodin significantly inhibited tumor growth. (B) The average body weight of individual groups of mice one week after the last treatment. (C) The average tumor diameter of individual groups of mice one week after the last treatment. * $\mathrm{P}<0.05$, compared to the control group.

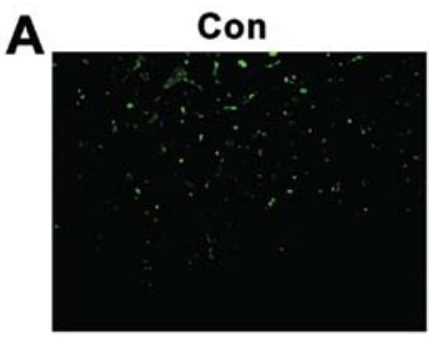

E40

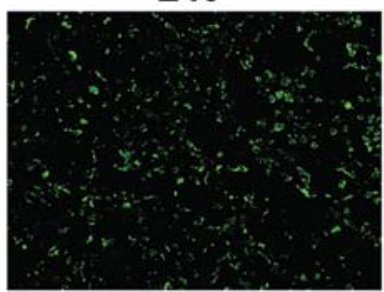

E10

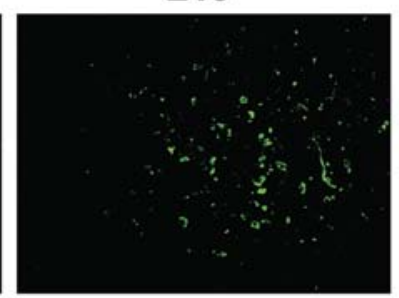

E80

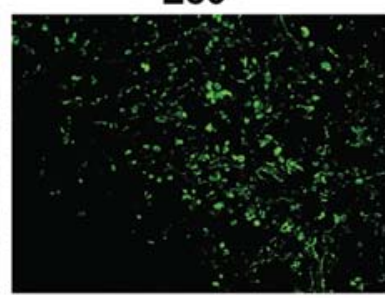

E20

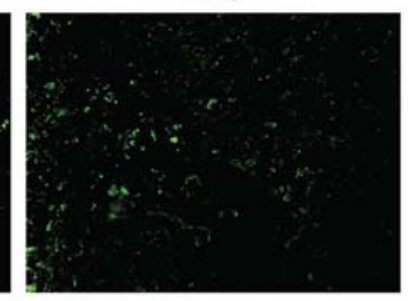

B

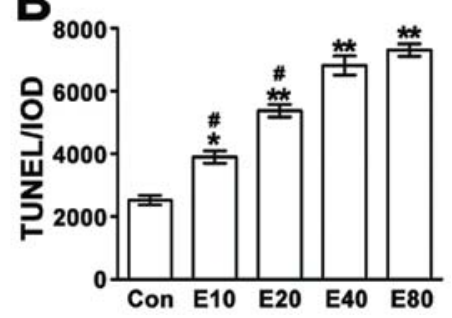

Figure 6. The effect of various concentrations of emodin on the apoptosis of implanted tumor cells. (A) Increased TUNEL-positive cells were found in the emodin treatment group (magnification, $\mathrm{x} 400$ ). TUNEL-positive cells of the tumor have an overlay of green fluorescence by the TUNEL staining. (B) TUNEL staining was further quantified and presented as average IOD level. ${ }^{*} \mathrm{P}<0.05$, compared to the control group; ${ }^{* *} \mathrm{P}<0.01$ compared to the control groups; ${ }^{~} \mathrm{P}<0.05$, compared to $\mathrm{E}_{40}$ group.

stuff intake of all groups decreased day by day. But the feeding stuff intake of $\mathrm{E}_{40}$ group declined less than the other groups. In the initial stage, feeding stuff intake of $\mathrm{E}_{80}$ group dramatically declined, but it changed gently in the last stage (Fig. 4).

Emodin inhibits the growth of tumor and increased the body weight of model mice. Compared to $\mathrm{E}_{40}$ and $\mathrm{E}_{80}$ groups, body weight in other groups was obviously reduced. The tumor diameter of $\mathrm{E}_{80}$ group was smaller than $\mathrm{E}_{40}$ group, but the body weight is smaller than $\mathrm{E}_{40}$ group (Fig. 5).

Emodin induces Panc-1 cell apoptosis in mouse tumors. Apoptosis of the tumor cells was detected by TUNEL assay. TUNEL-positive cells were observed with laser scanning confocal microscope (magnification, x400) (Fig. 6). With the increasing dose of emodin, the cell apoptosis increased. Apoptosis of tumor cells in $\mathrm{E}_{40}$ group and $\mathrm{E}_{80}$ group was significantly increased compared to the control group $(\mathrm{P}<0.01)$.

\section{Discussion}

Traditional Chinese medicine (TCM) used for tretment of cancer still have several controversies, unclear treatment mechanism and lack of theoretical basis of combination therapy $(7,8)$. Up to now, only few kinds of TCM are accredited and applied in international society. Even so, a survey report shows that approximately $40 \%$ of American cancer patients take TCM (9).

In recent years, more and more Chinese medicine monomers are extracted. These drugs are of high purity, with single 
chemical properties, such as emodin. Emodin is a kind of kinase inhibitor II (10), which could combine with DNA and prevent the proliferation and differentiation of tumor cells (11). Previous studies suggest that emodin may downregulate the nuclear factor- $\kappa \mathrm{B}(\mathrm{NF}-\kappa \mathrm{B})$ that could improve the proliferation, inhibit apoptosis, enhance cancer cell drug-resistance (12) and decrease the Bax/Bcl-2 ratio (13).

Mitochondria is not only the cells' energy factory, but also a 'suicide weapon' store because multiple apoptosis pathways stay there. Changing the tumor cells' metabolism of mitochondria or stimulating the MMP decline can significantly improve the efficiency of the cancer treatment (14). Mitochondria disorders are proved to be an important part of cell apoptosis, leading to many physiological changes (15). For example, the MMP changes early when the cell is interfered with by the apoptosis factor. The inner mitochondrial membrane permeabilization causes disruption of MMP (16). In recent years, many experiments suggest that keeping the MMP steady could prevent cells from tending to apoptosis (17) and once the MMP dissipates, the cell apoptosis will be irreversible (18). Keeping MMP normal is necessary for mitochondrial function. The decline of MMP is associated with the mitochondrial membrane permeability (MPT) changing, and MPT results from the opening of a mitochondrial permeability transition pore, also known as the MPT pore or MPTP (a protein pore that is formed in the inner membrane of the mitochondria). MPTP allows water and solutes to come into the matrix, causing the expansion of the mitochondria which results in the rupture of outer mitochondrial membrane (MOM) so that the apoptosis factors such as cyt c, Smac/DIABLO, AIF, release to cytoplasm, finally leading to cell apoptosis $(19,20)$. Our study showed that even a low concentration of emodin led to MMP decline. As the drug concentration increased, the MMP declined, with the cell apoptosis rate and cell proliferation inhibition rate rising. Our results suggest that emodin could induce MMP decline in Panc-1 cells, thereby leading to cell apoptosis and cell proliferation inhibition.

Gemcitabine was used to treat advanced pancreatic cancer as a standard drug. Most patients develop resistance to gemcitabine (21), which has strong toxic side effect and is very expensive. The success of this treatment is poor and overall survival has not improved for decades (22). In recent years, much attention was paid to Chinese traditional medicine monomers (CTMM). CTMM promotes apoptosis, inhibits proliferation, reverses drug-resistance in chemotherapy and improves the effect of chemotherapy on cancer therapy (23). Emodin is one of these drugs which has been shown to play a therapeutic role in the gastrointestinal tract, and genitourinary cancer (5,24-26). Our study showed that emodin suppressed tumor growth and induced tissue cell apoptosis. Emodin at the dose of $80 \mathrm{mg} / \mathrm{kg}$ still did not show strong drug toxicity as no unceasing decline of appetite of mice in this group was seen. At the end the body weight was more than control, $\mathrm{E}_{10}$ and $\mathrm{E}_{20}$ groups demonstrating that emodin may inhibit tumor growth and the mice can tolerate the drug toxicity.

In conclusion, our study suggests that emodin has a good effect on declining the MMP of Panc-1 cells, promoting apop- tosis and inhibiting cell proliferation. This drug improved the life quality of the mice with implanted tumors.

\section{Acknowledgments}

We are grateful for the funding support from the Administration of Traditional Chinese Medicine of Zhengjing Province, China (grant no. 2011ZZ010); the Zhejiang Provincial Science Fund for Distinguished Young Scholars (grant no. LR12H280001) and the National Natural Science Foundation of China (grant no. 81173606). We thank the entire staff of the Animal Experimental Center in Wenzhou Medical College and the scientific research platform of the Second Afiliated Hospital of Wenzhou Medical College for helpful assistance.

\section{References}

1. Maisonneuve $\mathrm{P}$ and Lowenfels AB: Epidemiology of pancreatic cancer: an update. Dig Dis 28: 645-656, 2010.

2. Triano LR, Chang BW and Saif MW: New developments in the treatment of locally advanced pancreatic cancer. Highlights from the 45th ASCO annual meeting. Orlando, FL. JOP 10: 366-372, 2009.

3. Conroy T, Desseigne F, Ychou M, et al: FOLFIRINOX versus gemcitabine for metastatic pancreatic cancer. N Engl J Med 364: 1817-1825, 2011.

4. Yu CX, Zhang XQ, Kang LD, et al: Emodin induces apoptosis in human prostate cancer cell LNCaP. Asian J Androl 10: 625-634, 2008.

5. Damodharan U, Ganesan R and Radhakrishnan UC: Expression of MMP2 and MMP9 (gelatinases A and B) in human colon cancer cells. Appl Biochem Biotechnol 165: 1245-1252, 2011.

6. Guo HC, Bu HQ, Luo J, et al: Emodin potentiates the antitumor effects of gemcitabine in PANC-1 pancreatic cancer xenograft model in vivo via inhibition of inhibitors of apoptosis. Int J Oncol 40: 1849-1857, 2012.

7. Baumann S, Fas SC, Giaisi M, et al: Wogonin preferentially kills malignant lymphocytes and suppresses T-cell tumor growth by inducing PLCgamma1- and $\mathrm{Ca}^{2+}$-dependent apoptosis. Blood 111: 2354-2363, 2008 .

8. Li S, Zhang B, Jiang D, Wei Y and Zhang N: Herb network construction and co-module analysis for uncovering the combination rule of traditional Chinese herbal formulae. BMC Bioinformatics 11: S6, 2010.

9. Oh B, Hu G, Kao S, Gebski V, Walls R, Truong L, Beale P and Clarke S: The safety and tolerability of Chinese herbal medicine in cancer patients receiving chemotherapy: pilot study. WebmedCentral Chinese Medicine 2: WMC001671, 2011.

10. Yim H, Lee YH, Lee CH and Lee SK: Emodin, an anthraquinone derivative isolated from the rhizomes of Rheum palmatum, selectively inhibits the activity of casein kinase II as a competitive inhibitor. Planta Med 65: 9-13, 1999.

11 Wang L, Lin L and Ye B: Electrochemical studies of the interaction of the anticancer herbal drug emodin with DNA. J Pharm Biomed Anal 42: 625-629, 2006.

12. Liu A, Chen H, Tong H, et al: Emodin potentiates the antitumor effects of gemcitabine in pancreatic cancer cells via inhibition of nuclear factor-kappaB. Mol Med Rep 4: 221-227, 2011.

13. Chen H, Wei W, Guo Y, et al: Enhanced effect of gemcitabine by emodin against pancreatic cancer in vivo via cytochrome C-regulated apoptosis. Oncol Rep 25: 1253-1261, 2011.

14. Fulda S, Galluzzi L and Kroemer G: Targeting mitochondria for cancer therapy. Nat Rev Drug Discov 9: 447-464, 2010.

15. Leber B, Lin J and Andrews DW: Still embedded together binding to membranes regulates Bcl-2 protein interactions. Oncogene 29: 5221-5230, 2010.

16. Yang CL, Ma YG, Xue YX, Liu YY,Xie H and Qiu GR: Curcumin induces small cell lung cancer NCI-H446 cell apoptosis via the reactive oxygen species-mediated mitochondrial pathway and not the cell death receptor pathway. DNA Cell Biol 31: 139-150, 2012.

17. Ly JD, Grubb DR and Lawen A: The mitochondrial membrane potential (deltapsi(m)) in apoptosis: an update. Apoptosis 8: 115-128, 2003. 
18. Tian X, Luo Y, Liu YP, et al: Downregulation of Bcl-2 and survivin expression and release of Smac/DIABLO involved in bufalin-induced HL-60 cell apoptosis. Zhonghua Xue Ye Xue Za Zhi 27: 21-24, 2006 (In Chinese).

19. Halestrap AP: Calcium, mitochondria and reperfusion injury: a pore way to die. Biochem Soc Trans 34: 232-237, 2006

20. Lopez M, Welsh K, Yuan H, et al: Antagonists of IAP-family anti-apoptotic proteins BTI. Probe Reports from the NIH Molecular Libraries Program, 2010.

21. Kim MP and Gallick GE: Gemcitabine resistance in pancreatic cancer: picking the key players. Clin Cancer Res 14: 1284-1285, 2008.

22. Long J, Zhang Y, Yu X, et al: Overcoming drug resistance in pancreatic cancer. Expert Opin Ther Targets 15: 817-828, 2011.
23. Tan W, Lu J, Huang M, et al: Anti-cancer natural products isolated from Chinese medicinal herbs. Chin Med 6: 27, 2011.

24. Wei WT, Chen H, Ni ZL, et al: Antitumor and apoptosispromoting properties of emodin, an anthraquinone derivative from Rheum officinale Baill, against pancreatic cancer in mice via inhibition of Akt activation. Int J Oncol 39: 1381-1390, 2011.

25. Wang QJ, Cai XB, Liu MH, Hu H, Tan XJ and Jing XB: Apoptosis induced by emodin is associated with alterations of intracellular acidification and reactive oxygen species in EC-109 cells. Biochem Cell Biol 88: 767-774, 2010.

26. Wang W, Sun Y, Li X, et al: Emodin potentiates the anticancer effect of cisplatin on gallbladder cancer cells through the generation of reactive oxygen species and the inhibition of survivin expression. Oncol Rep 26: 1143-1148, 2011. 